\title{
High mobility group box 1 is a novel substrate of dipeptidyl peptidase-IV
}

\author{
C. Marchetti • A. Di Carlo • F. Facchiano • C. Senatore • \\ R. De Cristofaro • A. Luzi • M. Federici • M. Romani • \\ M. Napolitano • M. C. Capogrossi • A. Germani
}

Received: 16 March 2011 / Accepted: 16 May 2011 /Published online: 8 June 2011

(C) Springer-Verlag 2011

\begin{abstract}
Aims/hypothesis High mobility group box 1 (HMGB1) is a cytokine with a key role in tissue regeneration and angiogenesis. Previous studies have shown that topical application of HMGB1 to skin wounds of mouse models of diabetes enhanced vessel density and accelerated wound healing, suggesting that diabetes may affect endogenous HMGB1 functions. Dipeptidyl peptidase IV (DPP-IV/
\end{abstract}

Electronic supplementary material The online version of this article (doi:10.1007/s00125-011-2213-6) contains peer-reviewed but unedited supplementary material, which is available to authorised users.

C. Marchetti · A. Di Carlo · M. Romani · M. Napolitano •

M. C. Capogrossi $\cdot$ A. Germani

Laboratorio di Patologia Vascolare,

Istituto Dermopatico dell'Immacolata,

Istituto di Ricovero e Cura a Carattere Scientifico,

Rome, Italy

F. Facchiano $\cdot$ C. Senatore

Department of Hematology, Oncology and Molecular Medicine, Istituto Superiore di Sanità,

Rome, Italy

R. De Cristofaro

Department of Internal Medicine \& Geriatrics,

Università Cattolica,

Rome, Italy

\section{A. Luzi $\cdot$ M. Federici}

Department of Internal Medicine, University of Rome Tor Vergata, Rome, Italy

\author{
A. Germani $(\bowtie)$ \\ Fondazione Livio Patrizi and Laboratorio di Patologia Vascolare, \\ Istituto Dermopatico dell'Immacolata, \\ Via dei Monti di Creta 104, \\ 00167, Rome, Italy \\ e-mail: a.germani@fondazioneliviopatrizi.eu
}

CD26) is a protease whose activity is increased in diabetes and whose inhibition improves glucose tolerance. Since HMGB1 contains potential DPP-IV cleavage sites, we determined whether HMGB1 may be a substrate for DPPIV and whether DPP-IV-mediated cleavage may alter the biological activity of HMGB1.

Methods Reversed phase HPLC, mass spectrometry and western blot analyses were performed to analyse and identify HMGB1 peptides generated following DPP-IV digestion. HMGB1 angiogenic functions in the presence of DPP-IV were evaluated in vitro and in vivo. HMGB1 protein was detected in the serum of type 2 diabetic patients before and after treatment with DPP-IV inhibitors.

Results DPP-IV cleaved HMGB1 at its N-terminal region and affected its angiogenic functions. Specifically, DPP-IV inhibited HMGB1-induced endothelial cell migration and capillary-like structure formation, as well as HMGB1mediated vascular network formation in Matrigel implants in mice. We had previously found that HMGB1 promoted endothelial cell migration through activation of extracellular regulated kinase signalling pathway. Here we showed that such an effect was abolished in the presence of DPP-IV. Finally, the N-terminal truncated form of HMGB1 was detected in the serum of type 2 diabetic patients, in whom DPP-IV inhibitors enhanced the levels of full-length HMGB1.

Conclusions/interpretation DPP-IV cleaves HMGB1 and, via this mechanism, inhibits HMGB1 angiogenic activity. Treatment with DPP-IV inhibitors may enhance HMGB1 activity in diabetic patients, thereby improving angiogenesis in this condition.

Keywords Basic science - Cells · Cytokines - Endothelium · Macrovascular complications - Oral pharmacological agents $\cdot$ Pathogenetic mechanisms 


$\begin{array}{ll}\text { Abbreviations } & \\ \text { DPP-IV } & \text { Dipeptidyl peptidase IV } \\ \text { EBM } & \text { Endothelial basal medium } \\ \text { EGM } & \text { Endothelial growth medium } \\ \text { EPC } & \text { Endothelial progenitor cells } \\ \text { ERK } & \text { Extracellular signal-regulated kinases } \\ \text { HMGB1 } & \text { High mobility group box 1 } \\ \text { HMGB1-pN- } & \text { Antibody directed against the HMGB1 } \\ \text { ter } & \text { N-terminal region } \\ \text { GIP } & \text { Glucose-dependent insulinotropic } \\ & \text { polypeptide } \\ \text { GLP } & \text { Glucagon-like peptide } \\ \text { ICAM-1 } & \text { Intercellular adhesion molecule 1 } \\ \text { MALDI } & \text { Matrix-assisted laser desorption/ionisation } \\ \text { MS } & \text { Mass spectrometry } \\ \text { SDF-1 } & \text { Stromal derived factor-1 } \\ \text { TOF } & \text { Time-of-flight } \\ \text { VCAM-1 } & \text { Vascular cell adhesion molecule 1 } \\ \text { VEGF } & \text { Vascular endothelial growth factor }\end{array}$

\section{Introduction}

Dipeptidyl peptidase IV (DPP-IV) is a $110 \mathrm{kDa}$ cell surface glycoprotein, which also exists as a soluble form circulating in plasma [1]. DPP-IV selectively removes the $\mathrm{N}$-terminal dipeptides with either L-proline or L-alanine at the penultimate position from a variety of chemokines, growth factors and neuropeptides, leading to their inactivation or degradation [2]. Glucose-dependent insulinotropic polypeptide (GIP) and glucagon-like peptide (GLP)-1 are endogenous physiological substrates for DPP-IV, and chemical inhibition of DPP-IV activity results in increased levels of intact bioactive GIP and GLP-1, and increased glucose tolerance. At present, DPP-IV inhibitors are used as glucose-lowering agents in the treatment of type 2 diabetes [3].

High mobility group box 1 protein (HMGB1) is a multifunctional ubiquitous protein that is released extracellularly in response to infection and injury. The extracellular HMGB1 protein signals tissue injury and evokes inflammatory and regenerative responses [4]. When added to endothelial cells, HMGB1 elicits proinflammatory responses by increasing the abundance of vascular adhesion molecules, as well as secretion of cytokines (TNF- $\alpha$ ) and chemokines (IL8 and monocyte chemoattractant protein-1) $[5,6]$. It also plays a role in angiogenesis-modulating functions of endothelial cells [79], endothelial progenitor cells (EPC) [10] and vesselassociated stem cells [11]. Specifically, HMGB1 induces endothelial cell migration, sprouting and neovascularisation $[7,8]$ through a mechanism involving activation of extracellular signal-regulated kinases (ERK) and c-Jun N- terminal kinase signalling [9]. In skin of diabetic patients, HMGB1 levels are reduced and its topical application to skin wounds of diabetic mice enhanced vessel density and accelerated wound healing [12].

Since HMGB1 contains potential DPP-IV cleavage sites, we determined (1) whether HMGB1 may be a substrate for DPP-IV and (2) whether DPP-IV-mediated cleavage may account for the altered angiogenic activity of HMGB1 in diabetes.

\section{Methods}

Cell culture and treatment HUVEC (Cambrex, Bergamo, Italy) were cultured in endothelial growth medium (EGM)2-containing growth factors (Cambrex). Cells were used between passage three and six. Treatments with HMGB1 (HMGBiotech, Milan, Italy) and DPP-IV (Sigma Aldrich, Como, Italy) were performed in EBM-2 (Cambrex). In all experiments, HMGB1 was pre-incubated at $37^{\circ} \mathrm{C}$ for $2 \mathrm{~h}$ in the presence or absence of DPP-IV, before being administered to the culture medium. For western blot analysis, cells were lysed in $10 \mathrm{mmol} / \mathrm{l}$ Tris- $\mathrm{HCl}$ (pH 7.4), $150 \mathrm{mmol} / \mathrm{l} \mathrm{NaCl}, 1 \%$ (vol./vol.) NP40, $1 \%$ (wt/vol.) deoxycholic acid, $0.1 \%$ (wt/vol.) SDS, $10 \%$ (vol./vo.l) glycerol and protease inhibitors. Membranes were probed with ERK1/2 and pERK antibodies (Cell Signaling Technology, Danvers, MA, USA), followed by horseradish peroxidase-coupled secondary antibodies, and images were developed by a chemiluminescence-based detection system (ECL; Amersham, Milan, Italy).

Cleavage of HMGB1 by DPP-IV HMGB1 $(5 \mu \mathrm{g})$ was incubated with DPP-IV $(0.5 \mu \mathrm{g})$ (Sigma Aldrich) for up to $24 \mathrm{~h}$ in $50 \mathrm{mmol} / 1 \mathrm{Tris}-\mathrm{HCl} \mathrm{pH} 7.4$ and $1 \mathrm{mmol} / \mathrm{l}$ EDTA. The supernatant fraction containing peptides was fractionated on an analytical column (ReproSil-Pur C18-AQ; Dr Meisch-GmBH, Ammerbuch, Germany), connected to a double-pump (model 2080 HPLC; Jasco, Tokyo, Japan). The eluted peptides were detected simultaneously by a UV/ VIS spectrophotometric device at $210 \mathrm{~nm}$ (model 2075; Jasco) and by a spectrofluorometric detector (FP-2020; Jasco) using $\lambda_{\text {exc }} 280 \mathrm{~nm}$ and a $\lambda_{\text {em }} 350 \mathrm{~nm}$. The quantification of the area of the chromatographic peak pertaining to the cleaved peptide was obtained using Borwin software (Jasco).

For matrix-assisted laser desorption/ionisation (MALDI) time-of-flight (TOF) studies the supernatant fraction obtained after incubation of HMGB1 with DPP-IV was loaded and analysed by Voyager-DE STR (Applied Biosystems, Carlsbad, CA, USA) with TOF/mass spectrometry (MS) operating in positive-ion linear mode. Spectra were analysed by Data Explorer (version 4.0.0.0; 
Applied Biosystems) and Moverz software $(\mathrm{m} / \mathrm{z}$-Knexus edition; Proteometrics, LLC, New York, NY, USA) [12]. For details, see electronic supplementary material (ESM), Cleavage of HMGB1.

Migration assays Migration was performed in chemotaxis chambers (Neuroprobe, Cabin John, MD, USA) using $8 \mathrm{~mm}$ pore size polycarbonate filters (Costar Scientific, Cambridge, MA, USA) coated with murine collagen type IV $(5 \mu \mathrm{g} / \mathrm{ml}$; Becton-Dickinson, Franklin Lakes, NJ, USA). Briefly, $0.7 \times 10^{6}$ cells $/ \mathrm{ml}$ were added to the upper chambers and the indicated factors were placed in the lower chambers. Migration was stopped after $4 \mathrm{~h}$. See ESM, Chemotaxis for details.

In vitro angiogenesis assay Reconstituted basement membrane proteins (Matrigel), growth factor-reduced (Collaborative Research Beckton Dickinson, Bedford, MA, USA), was applied to 24 -well culture plates. HUVEC $\left(8 \times 10^{4}\right.$ in $1 \mathrm{ml}$ medium) were seeded on Matrigel-coated wells in the presence of EBM-2 medium supplemented with the indicated factors and kept at $37^{\circ} \mathrm{C}$ to allow differentiation to occur. The presence of tubular structures was determined $5 \mathrm{~h}$ after plating by phase-contrast microscopy. Quantification of tubular structure formation was expressed by the mean number of branching points in five fields. Experiments were carried out three times in duplicate.

In vivo angiogenesis assay Matrigel $(400 \mu \mathrm{l})$ (Becton Dickinson) was mixed with HMGB1 $(400 \mathrm{ng} / \mathrm{ml})$, DPPIV $(40 \mathrm{ng} / \mathrm{ml})$ and Diprotin A $(5 \mu \mathrm{mol} / \mathrm{l})$ alone or in combination and injected subcutaneously in CD1 male mice (Charles River, Calco, Italy). At 8 days after Matrigel injection, plugs were removed and processed for histological analysis as previously described [13]. All experimental procedures complied with the Guidelines of the Italian National Institutes of Health and the Guide for the Care and Use of Laboratory Animals (Institute of Laboratory Animal Resources, National Academy of Sciences, Bethesda, MD, USA), and were approved by the Institutional Animal Care and Use Committee.

Patients Blood samples were collected from normoglycaemic healthy donors $(n=10)$ matched for age, sex and BMI (six men, four women; age $55 \pm 2.0$ years, BMI $30 \pm 0.9 \mathrm{~kg} / \mathrm{m}^{2}$, $\left.\mathrm{HbA}_{\mathrm{lc}} 5.4 \pm 0.1 \%[36 \pm 1.2 \mathrm{mmol} / \mathrm{mol}]\right)$ and from type 2 diabetic patients $(n=28,17$ men, 11 women; age 59.9 \pm 1.7 , BMI $29.9 \pm 0.85 \mathrm{~kg} / \mathrm{m}^{2}, \quad \mathrm{HbA}_{\mathrm{lc}} 7.3 \pm 0.15 \%$ [52.2 \pm $1.1 \mathrm{mmol} / \mathrm{mol}])$. All diabetic participants were being treated with metformin as oral hypoglycaemic agent. Among these, 13 were also treated with sitagliptin or vitagliptin (100 mg per day) for 1 month. This research study was carried out in accordance with the Declaration of Helsinki (2000) of the World Medical Association and was approved by the Ethics Committee of the University of Rome Tor Vergata.

Assay for DPP-IV activity DPP-IV activity in the serum of non-diabetic and diabetic patients was measured using the chromogenic substrate Gly-Pro- $p$-nitroanilide (GlyPro-pNA) (Sigma Aldrich) according to the manufacturer's instructions.

Proteolytic activity was determined by measuring the amount of nitroanilide (pNA) formed in the supernatant fraction at $405 \mathrm{~nm}$. Activity is expressed as picomoles/ minute (U) per 1,000 cells. Inhibition of DPP-IV was accomplished by treatment of cells with $5 \mathrm{mmol} / 1$ of a DPPIV inhibitor called Diprotin A, a three-amino-acid peptide (Ile-Pro-Ile) (Sigma Aldrich).

HMGB1 immunoassay HMGB1 levels were measured using an ELISA assay (Shino-Test Corporation, Kanagawa, Japan) according to the manufacturer's instructions.

Data collection and statistics Data are expressed as mean \pm SE. Significant differences between groups of data were assessed using the unpaired Student's $t$ test. A $p$ value of $p<$ 0.05 was considered statistically significant.

\section{Results}

$H M G B 1$ cleavage by DPP-IV To investigate whether HMGB1 is cleaved by DPP-IV, $5 \mu \mathrm{g}$ recombinant HMGB1 were incubated with $0.5 \mu \mathrm{g}$ of the purified enzyme for increasing periods of time and cleaved peptides then analysed by reversed-phase HPLC (Fig. 1a-d). The analysis revealed a peptide whose concentration expressed as peak area increased as a function of time (Fig. 1a-c). Addition of the DPP-IV inhibitor Diprotin A in the $24 \mathrm{~h}$ reaction mixture partially reduced the peak area at this time point (Fig. 1d).

MALDI-TOF/MS analysis allowed detection of several ion masses in HMGB1 samples (Fig. 1e, f). Of these, one fragment with $23,810.86 \mathrm{~m} / \mathrm{z}$ value was detected only in the sample in which HMGB1 was incubated with DPP-IV (Fig. 1e). This fragment represented a truncated protein derived from the native one lacking a peptide with 973.11 mass $(\mathrm{m} / \mathrm{z})$. Bioinformatic analyses by Data Explorer and ASC [14] suggested that such a mass difference is likely to correspond to the N-terminal peptide of HMGB1 (MGKGDPKKP, calculated molecular mass $974.16 \mathrm{Da}$ ) (Fig. 1e).

The DPP-IV-mediated deletion of the N-terminal region of HMGB1 was further confirmed by western blot analysis of the HMGB1/DPP-IV mixture: time-dependent disap- 

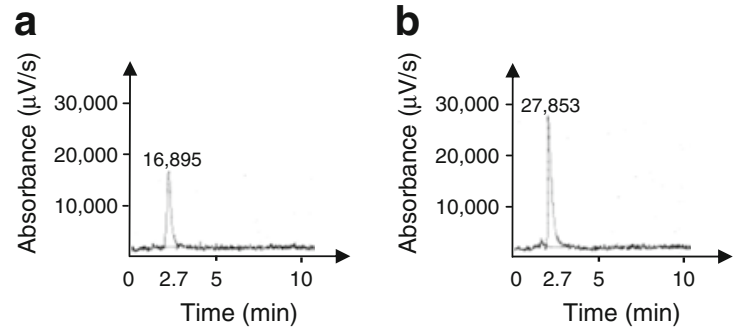

e

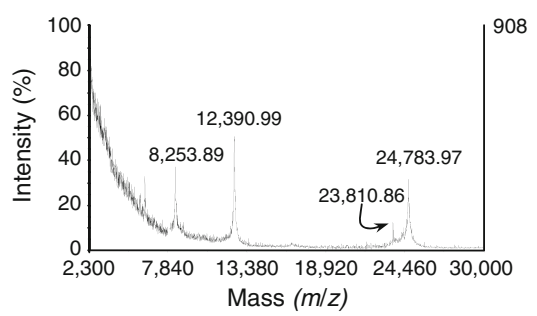

g

$\mathrm{ab} 67281$

MGKGDPKKPRGKMSSYAFFVQTCREEHKKKHPDASVNFSEFSKKC SERWKTMSAKEKGKFEDMAKADKAREREMKTYIPPKGETKKKFKDP NAPKRPPSAFFLFCSEYRPKIKGEHPGLSIGDVAKKLGEMWNNTAAD DKQPYEKKAAKLKEKYEKDIAAYRAKGKPDAKKGVVKAEKSKKKKEE EEDEEDEEDEEEEEDEEDEDEEEDDDDD

Fig. 1 Purified DPP-IV cleaves HMGB1. a Representative HPLC profiles obtained after incubation of HMGB1 with DPP-IV for 2, (b) 4 and (c) $24 \mathrm{~h}$. A peak with a retention time of $2.7 \mathrm{~min}$ was present in all HMGB1 samples containing DPP-IV and was reduced when the DPPIV inhibitor Diprotin A was added to the HMGB1 and DPP-IV mixture (d). The $y$-axis shows the absorbance, measurement of current that correlates with peak intensity. e, f Representative MALDI-TOF/MS analysis of HMGB1. Peaks are labelled with their molecular mass; the peak of $23,810.86$ was present (e) in the HMGB1+DPP-IV sample,

pearance of the molecular mass band corresponding to HMGB1 was detected with an anti-HMGB1 antibody that recognises the first $16 \mathrm{~N}$-terminal amino acids of HMGB1 (Fig. 1g, h).

DPP-IV inhibits HMGB1-induced endothelial cell migration and tubular-like structure formation Migration is a key process required for angiogenesis and HMGB1 migratory activity on endothelial cells $[7,8]$. Therefore we investigated whether DPP-IV affected HMGB1-mediated endothelial cell migration in a multiwall chemotaxis chamber. Under the experimental conditions of the present study, HMGB1 exerted a migratory activity on HUVEC that, at a concentration of $200 \mathrm{ng} / \mathrm{ml}$, was similar to that induced by the complete medium (Fig. 2a). The HMGB1-mediated effect was abolished in the presence of $10 \mathrm{ng}$ DPP-IV, while a lowest dose of $1 \mathrm{ng}$ DPP-IV did not significantly modify HMGB1 migratory activity (Fig. 2a).
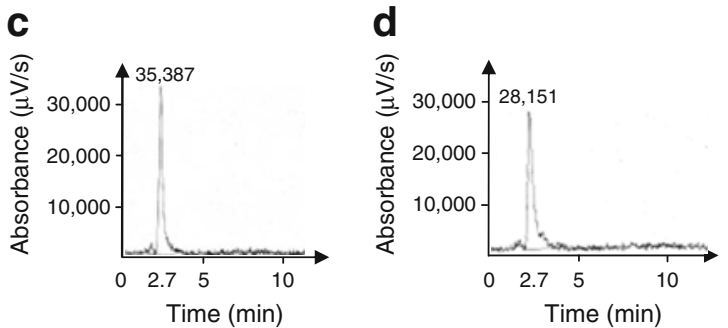

\section{$f$}

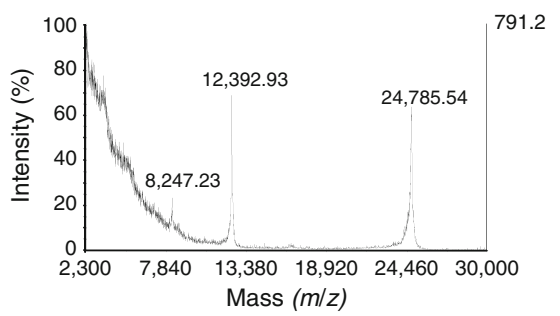

h

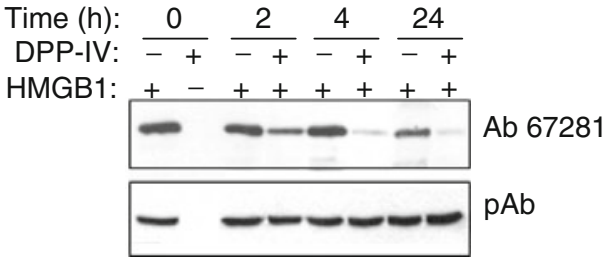

but was not detected in the absence of DPP-IV (f). The reported $\mathrm{m} / \mathrm{z}$ values are representative of one experiment repeated three times with similar results. g HMGB1 protein sequence, with peptide recognised by indicated antibody marked by box. $\mathbf{h}$ Western blot analysis of HMGB1 $(5 \mu \mathrm{g})$ incubated with DPP-IV $(0.5 \mu \mathrm{g})$ for the indicated time points. The same filter was probed with the anti-HMGB1 antibody AB67281 that recognises the $\mathrm{N}$-terminal portion of the protein and with a polyclonal anti-HMGB1 antibody (pAb)

HUVECs, when seeded on Matrigel in the presence of angiogenic growth factors, become elongated and form tubular-like structures, mimicking the in vivo neoangiogenesis process. As shown in Fig. 2b, HUVECs at $6 \mathrm{~h}$ after seeding exhibited a clear network formation in the presence of HMGB1, an effect that $10 \mathrm{ng}$ DPP-IV dramatically inhibited (Fig. 2b).

In in vivo studies, Matrigel supplemented with HMGB1 and DPP-IV, alone or in combination, was injected subcutaneously into the mid-lower abdominal region of $\mathrm{C} 57$ mice. In this model, host endothelial cells and smooth muscle cells migrate and form a vascular network in the Matrigel implants. Quantitative analysis of the Matrigel plugs at 8 days after implantation revealed a higher number of vessels in the plugs containing HMGB1 than in control $(37.3 \pm 6$ vs $11.1 \pm$ 4.2) (Fig. 2c). Vessel number was significantly reduced when HMGB1 was administered together with DPP-IV 

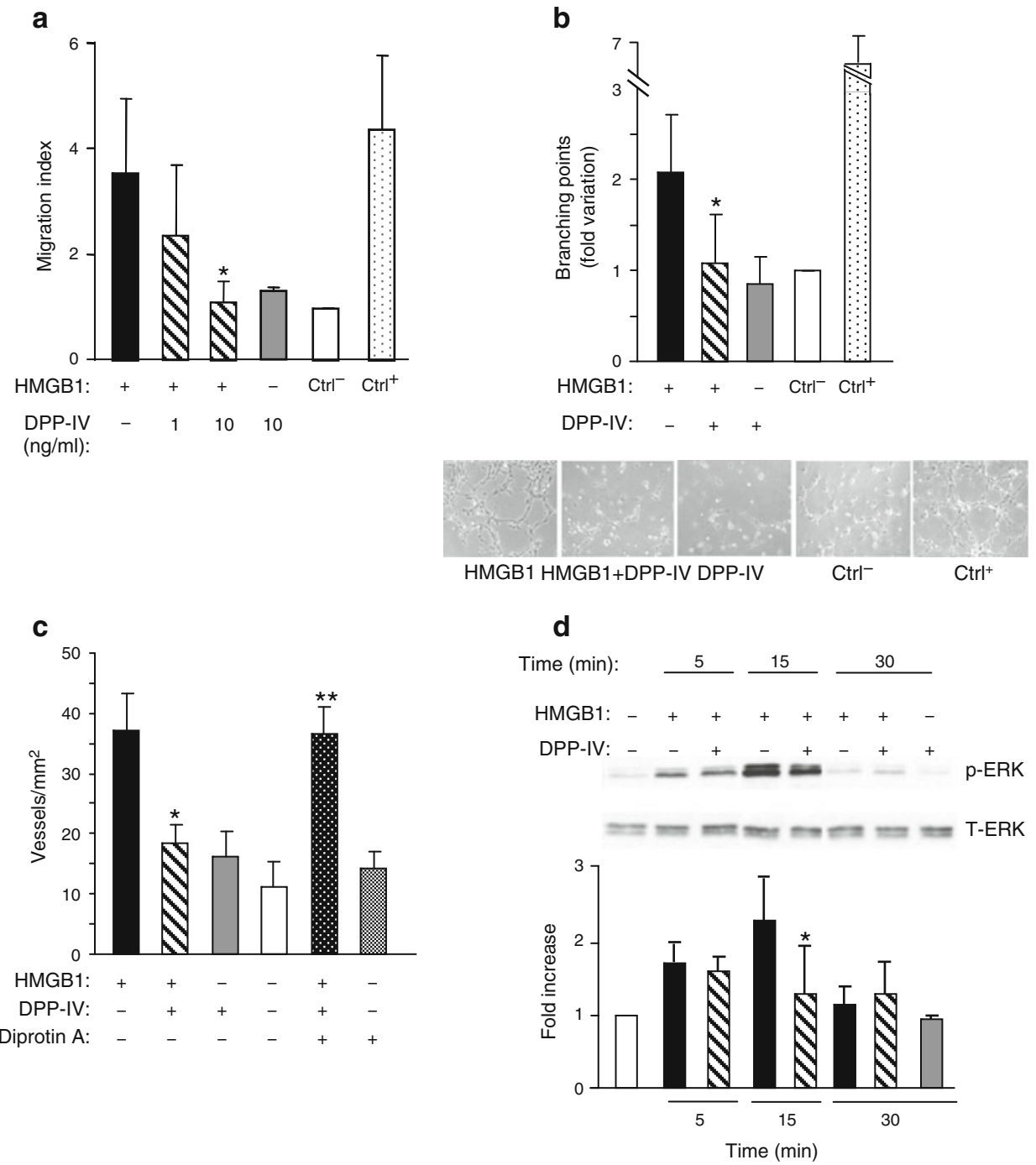

Fig. 2 DPP-IV inhibits HMGB1-induced endothelial function. a DPPIV inhibits HMGB1-induced endothelial cell migration. HUVECs were subjected to migratory assays with HMGB1 $(200 \mathrm{ng} / \mathrm{ml})$ in the absence or presence of DPP-IV (1 and $10 \mathrm{ng} / \mathrm{ml}$ ). EGM and EBM were used as positive $(\mathrm{Ctrl}+)$ and negative $(\mathrm{Ctrl}-)$ controls, respectively. Results are reported as mean $\pm \mathrm{SE}$ of four independent experiments. $* p<0.05$ vs HMGB1 alone. b DPP-IV inhibits HMGB1-induced tubular-like structure formation by endothelial cells. HUVECs were cultured on Matrigel in absence or in presence of HMGB1 (200 ng/ml) and DPP-IV (10 ng/ml). After $4 \mathrm{~h}$, branching points were counted over five representative fields for each well. Results reported are mean $\pm \mathrm{SE}$ of four independent experiments performed in duplicate. ${ }^{*} p<0.05$ vs HMGB1 alone. Representative photomicrographs show HUVECs plated on Matrigel in the indicated

(18.4 \pm 3.1$)$ and was similar to that present in saline imbibed Matrigel. Notably, the addition of Diprotin A, together with HMGB1 and DPP-IV, reversed the DPP-IV inhibitory effect on HMGB1-induced vessel formation (Fig. 2c).

In a previous study we showed that HMGB1 angiogenic activity was mediated by ERK phosphorylation. When HUVECs were incubated with HMGB1 alone, ERK

culture conditions. $\mathbf{c}$ Effect of DPP-IV on the angiogenic response to HMGB1 in Matrigel plugs in vivo. HMGB1 alone (400 ng) or in combination with DPP-IV $(10 \mathrm{ng} / \mathrm{ml})$ was added to Matrigel before subcutaneous injection in mice. Total number of neovessels over whole area of Matrigel was measured after 8 days. Results reported are mean $\pm \mathrm{SE}$ of five independent experiments. ${ }^{*} p<0.05$ and ${ }^{* *} p<$ 0.01 vs HMGB1 alone and HMGB1 plus DPP-IV plugs, respectively. d Western blot analysis of total extract from HUVECs cultured for the indicated time with HMGB1 in the absence or presence of DPP-IV. The filter was probed with anti phosphor-ERK (p-ERK). Normalisation of the lanes was performed using an antibody directed against total ERK1/2 (T-ERK). Findings were quantified by densitometric analysis and are shown as average results; $n=3$. ${ }^{*} p<0.05$ vs HMGB1 treated cells for the same time

phosphorylation increased between 5 and $15 \mathrm{~min}$, returning to basal levels after $30 \mathrm{~min}$ of HMGB1 stimulation. In the presence of DPP-IV, a significant reduction of ERK phosphorylation was observed in HUVECs stimulated at 15 min (Fig. 2d).

DPP-IV and HMGB1 in type 2 diabetic patients Several studies have demonstrated that DPP-IV activity is increased 
in the plasma of type 2 diabetic patients $[15,16]$. We confirmed that DPP-IV activity was higher in type 2 diabetic patients than in normoglycaemic counterparts (Fig. 3a). Interestingly, DPP-IV activity was also enhanced in high glucose-cultured endothelial cells (Fig. 3b). HMGB1 levels slightly but significantly increased in type 2 diabetic serum compared with normoglycaemic controls (Fig. 3c). We also assessed HMGB1 levels and DPP-IV activity in type 2 diabetes before and after treatment with DPP-IV inhibitors, i.e. gliptins. Of 28 patients enrolled and treated with a stable dose of metformin, 13 were randomly assigned to receive gliptins and 15 patients were treated with placebo. Plasma samples were collected before and 1 month after treatment. As expected, DPP-IV activity was significantly reduced in gliptin-treated patients (Fig. 3d) and a trend for HMGB1 plasma levels to increase was also detected (ESM Fig. 1a). In the placebo group, neither HMGB1 levels nor DPP-IV activity were affected (ESM Fig. 1a, b). Since DPP-IV cleaved HMGB1 in vitro, we investigated whether plasma HMGB1 in type 2 diabetic patients lacked its $\mathrm{N}$-terminal region and also whether a

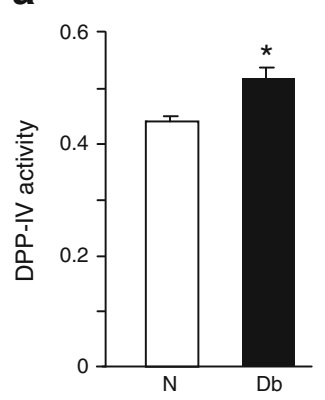

C

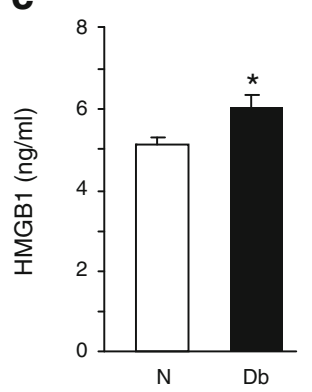

b

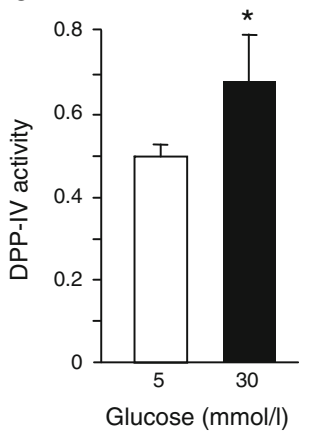

d

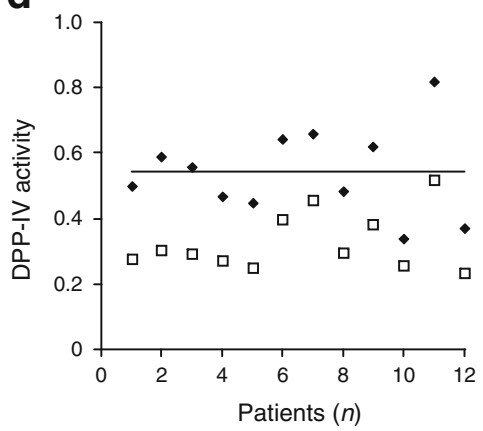

Fig. 3 DPP-IV inhibition and HMGB1 levels in vivo. DPP-IV activity in (a) plasma of normoglycaemic $(\mathrm{N})$ and type 2 diabetic $(\mathrm{Db})$ patients ( $n=13$ patients per group), and (b) in HUVECs cultured in low $(5 \mathrm{mmol} / \mathrm{l})$ and high $(30 \mathrm{mmol} / \mathrm{l})$ glucose $(n=4)$. c HMGB1 levels in plasma of the same patients as above (a). d DPP-IV activity in plasma of patients before (black diamonds) and 1 month after (white squares) gliptin treatment $\left(n=13\right.$ patients, $\left.R^{2}=2 \times 10^{-6}\right)$. * $p<0.05$. Pearson's method was used for the assessment of correlations. The regression line is shown as a horizontal line gliptin treatment prevented HMGB1 cleavage. The HMGB1 polyclonal antibody was used to immunoprecipitate similar amounts of HMGB1 from plasma samples of normoglycaemic and type 2 diabetic patients before and 1 month after gliptin treatment. Samples were analysed by western blot probing filters using HMGB1 polyclonal antibody and the antibody directed against the HMGB1 $\mathrm{N}$-terminal region (HMGB1-pN-ter). HMGB1 polyclonal antibody detected HMGB1 in normoglycaemic and in type 2 diabetic patient plasma. However, when the same blot was probed with HMGB1-pN-ter, HMGB1 was detectable only in normoglycaemic (Fig. 4a) and in gliptin-treated type 2 diabetic plasma (Fig. 4b). Together these data demonstrate that DPP-IV inhibition in vivo increases the levels of HMGB1 uncleaved form.

\section{Discussion}

In this report we provide evidence that DPP-IV cleaves HMGB1 at its N-terminal region. In addition, we demonstrated that DPP-IV markedly reduces HMGB1-induced endothelial cell migration and tubular-like structure formation in vitro, as well as vessel formation in vivo in the Matrigel plug assay. Our data support a recent observation by Takasawa showing that inhibition of DPP-IV enzymatic activity may affect endothelial cell functions, including endothelial cell proliferation and ex vivo neovascularisation following TNF- $\alpha$ or IL-1 $\beta$ stimulation [17].

The impairment of angiogenesis in diabetes is well documented and diminished peripheral blood flow, as well
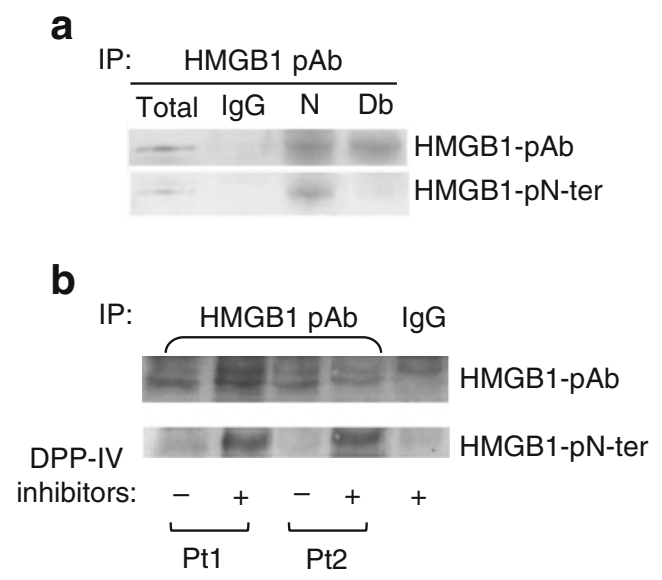

Fig. 4 DPP-IV cleaves HMGB1 in vivo. a HMGB1 immunoprecipitation (IP) from plasma of normoglycaemic (N) and type 2 diabetic (Db) patients, and (b) from two patients (Pt1 and Pt2) with type 2 diabetes before $(-)$ and after $(+)$ gliptin treatment. The same filter was probed with the anti-HMGB1 antibody AB67281 that recognises the $\mathrm{N}$-terminal portion of the protein (HMGB1-pN-ter) and with a polyclonal anti-HMGB1 antibody (HMGB1-pAb) 
as decreased local neovascularisation, are critical factors contributing to common vascular complications in diabetes such as chronic non-healing wounds and hindlimb ischaemia [18]. Although biological mechanisms involved in diabetes-related vascular defects so far have remained elusive, growth factor reduction and/or inactivation, as well as increased production of angiogenesis inhibitors, may account for these defects [18]. In diabetic mouse models of skin wound and in hindlimb ischaemia a reduction of vascular endothelial growth factor (VEGF), stromal derived factor-1 (SDF-1), HMGB1 and other growth factor abundance and/or activity has been documented [17]. Moreover, we previously reported that topical application of HMGB1 to skin wounds enhanced wound healing and angiogenesis in diabetic, but not in normoglycaemic mice [17]. Similarly, HMGB1 administration to ischaemic hindlimb of diabetic mice restored collateral blood flow. Notably this process was associated with increased levels of VEGF [17].

The degradation and/or inactivation of soluble factors involved in angiogenesis may in part be due to increased proteolytic activity in the serum and in tissues of diabetic patients. The protease DPP-IV may be one such key regulatory molecule. Consistent with this hypothesis, DPP-IV levels increased in high glucose-cultured microvascular endothelial cells $[19,20]$, as well as in primary endothelial cells under similar culture conditions, as detected here. Although the evaluation of DPP-IV levels in plasma of type 2 diabetic patients in comparison with matched healthy controls has yielded controversial results $[15,16,21,22]$, we found here that DPP-IV activity was enhanced in the plasma of type 2 diabetes patients. Importantly, although HMGB1 levels were slightly higher in diabetic than in normoglycaemic participants, the circulating form of the protein lacked its $\mathrm{N}$-terminal region. Treatment with gliptins for 1 month prevented the DPP-IVmediated cleavage of HMGB1. Based on our results, we therefore suggest that DPP-IV inhibitors may enhance HMGB1 function not only in vitro but also in diabetic patients.

It is noteworthy that HMGB1 is not the only target molecule of DPP-IV to have an angiogenic activity. The chemokines SDF- $1 \alpha$ and SDF- $\beta$ are cleaved and inactivated by DPP-IV at the NH2-terminus, blocking their ability to activate the chemokine (C-X-C motif) receptor 4 [23, 24]. Similarly to HMGB1, SDF-1 has been involved in angiogenesis, modulating endothelial cell function, and inducing EPC mobilisation and recruitment to the site of ischaemic injury [25]. Notably, EPCs are key cellular effectors of postnatal neovascularisation. Their circulating numbers are decreased in diabetes, which, together with the functional reduction of growth factors involved in their mobilisation and recruitment, has been implicated in the altered vessel growth in diabetic conditions. There- fore, by lowering SDF-1 and HMGB1 catabolism, DPPIV inhibition could represent a promising strategy to improve vessel growth directly and by stimulating EPC homing. Accordingly, in a mouse model of myocardial infarction, DPP-IV inhibition improved cardiovascular outcomes [26] as well as tissue vascularisation enhancing cardiac homing of stem cells [27], while in diabetic patients, the DPP-IV inhibitor sitagliptin increased circulating EPCs and SDF-1 levels [28].

Several points need to be addressed in future studies. We evaluated here the effect of DPP-IV and its inhibition of HMGB1 angiogenic function. HMGB1 is known to induce a proinflammatory phenotype in endothelial cells in vitro through upregulation of leucocyte adhesion molecules (intercellular adhesion molecule 1 [ICAM-1] and vascular cell adhesion molecule 1 [VCAM-1]) $[5,6]$. Therefore, it is possible that DPP-IV inhibitors further enhance the proinflammatory role of HMGB1 in vitro and in vivo. Since endothelial dysfunction in diabetes is associated with increased levels of circulating soluble adhesion molecules, including ICAM-1 and VCAM-1 [29, 30], DPP-IV inhibitors may further enhance serum levels of these molecules, preserving HMGB1 proinflammatory functions.

Although we showed that HMGB1 is a substrate of DPP-IV in vitro, it should be considered that other DPP-IV peptidases may be involved in HMGB1 cleavage in vivo [31]. Among them, DPP8 and DPP9 possess DPP-IV-like enzyme activity and are produced in microvascular and macrovascular endothelial cells as soluble enzymes [32]. DPP8 and DPP9 cleave DPP-IV substrates GLP-1, GLP-2 and neuropeptide $Y$ [33]. More recently, it has been shown that SDF- $1 \alpha$ and SDF- $\beta$ are hydrolysed to a similar extent by DPP-IV and DPP8 [33, 34].

Intriguingly, therefore, DPP8 and/or DPP9 may contribute to some of the functions ascribed to DPP-IV. Finally, since we measured DPP-IV activity in plasma of diabetic patients using a common substrate for DPP-IV, DPP8 and DPP9, and considering that vildagliptin and, to a lesser extent, sitagliptin may also inhibit both DPP8 and DPP9 [31], we cannot exclude the possibility that the DPP-IV activity measured in plasma before and after gliptin treatment was only due to DPP-IV activity. Finally, the involvement of DPP-IV inhibition in diabetic retinopathy should also be addressed. This complication has been associated with increased levels of angiogenic factors VEGF and SDF-1 in the vitreous, which in turn promote intra-ocular neovascularisation [35, 36]. Although HMGB1 levels in the vitreous have not been assessed, DPP-IV inhibitors could enhance the active form of vitreal SDF-1, potentially worsening diabetic retinopathy.

To clarify these aspects, we suggest that the pharmacological inactivation of the DPP-IV family of enzymes may contribute not only to improvement of glucose tolerance in 
diabetic patients, but may also affect diabetes-associated vascular complications.

Acknowledgements We thank A. Musarò of the Department of Histology and Medical Embryology, Sapienza University of Rome, and the Telethon Proteomics Service at Istituto Superiore di Sanità, Rome. We also thank C. Capuzzello of the Istituto Dermopatico dell'Immacolata for experimental suggestions. This work was supported by Fondazione Roma and the Italian Ministry of Health. C. Senatore was involved in the training programme at the $\mathrm{PhD}$ School of Human Pathology, Department of Experimental Medicine, Sapienza University of Rome, Rome, Italy.

C. M. and A. Di C. were responsible for the conception and design of the study and revised the article critically; F. F., C. S., R. De C. and A. L. were responsible for the conception and design of the study, moreover they revised the manuscript; M. R. was responsible for the conception and design of the study and for revising the manuscript; $\mathrm{M}$. F. was responsible for the analysis and interpretation of data and for revising the manuscript; M. N. and M. C. C. were responsible for conception of the study and for revising the article critically. A. G. was responsible for the analysis and interpretation of data, design of the article and drafting the article. All authors gave final approval.

Duality of interest The authors declare that there is no duality of interest associated with this manuscript.

\section{References}

1. Durinx C, Lambeir AM, Bosmans E et al (2000) Molecular characterization of dipeptidyl peptidase activity in serum: soluble CD26/dipeptidyl peptidase IV is responsible for the release of XPro dipeptides. Eur J Biochem 267:5608-5613

2. Yazbeck R, Howarth GS, Abbott CA (2009) Dipeptidyl peptidase inhibitors, an emerging drug class for inflammatory disease? Trends Pharmacol Sci 30:600-607

3. Nauck MA (2011) Incretin-based therapies for type 2 diabetes mellitus: properties, functions, and clinical implications. Am J Med 124(1 Suppl):S3-S18

4. Klune JR, Dhupar R, Cardinal J et al (2008) HMGB1: endogenous danger signaling. Mol Med 14:476-484

5. Treutiger CJ, Mullins GE, Johansson AS et al (2003) High mobility group 1 B-box mediates activation of human endothelium. J Intern Med 254:375-385

6. Fiuza C, Bustin M, Talwar S et al (2003) Inflammation-promoting activity of HMGB1 on human microvascular endothelial cells. Blood 101:2652-2660

7. Mitola S, Belleri M, Urbinati C et al (2006) Cutting edge: extracellular high mobility group box-1 protein is a proangiogenic cytokine. J Immunol 176:12-15

8. Schlueter C, Weber H, Meyer B et al (2005) Angiogenetic signaling through hypoxia: HMGB1: an angiogenetic switch molecule. Am J Pathol 166:1259-1263

9. De Mori R, Straino S, Di Carlo A et al (2007) Multiple effects of high mobility group box protein 1 in skeletal muscle regeneration. Arterioscler Thromb Vasc Biol 27:2377-2383

10. Chavakis E, Hain A, Vinci M et al (2007) High-mobility group box 1 activates integrin-dependent homing of endothelial progenitor cells. Circ Res 100:204-212

11. Palumbo R, Sampaolesi M, de Marchis F et al (2004) Extracellular HMGB1, a signal of tissue damage, induces mesoangioblast migration and proliferation. J Cell Biol 164:441-449
12. Straino S, Di Carlo A, Mangoni A et al (2008) High-mobility group box 1 protein in human and murine skin: involvement in wound healing. J Invest Dermatol 128:1545-1553

13. Straino S, Germani A, Di Carlo A et al (2004) Enhanced arteriogenesis and wound repair in dystrophin-deficient $\mathrm{mdx}$ mice. Circulation 110:3341-3348

14. Facchiano AM, Facchiano A, Facchiano F (2003) Active Sequences Collection (ASC) database: a new tool to assign functions to protein sequences. Nucleic Acids Res 31:379-382

15. Mannucci E, Pala L, Ciani S et al (2005) Hyperglycaemia increases dipeptidyl peptidase IV activity in diabetes mellitus. Diabetologia 48:1168-1172

16. Ryskjaer J, Deacon CF, Carr RD et al (2006) Plasma dipeptidyl peptidase-IV activity in patients with type- 2 diabetes mellitus correlates positively with HbAlc levels, but is not acutely affected by food intake. Eur J Endocrinol 155:485-493

17. Takasawa W, Ohnuma K, Hatano R et al (2010) Inhibition of dipeptidyl peptidase 4 regulates microvascular endothelial growth induced by inflammatory cytokines. Biochem Biophys Res Commun 401:7-12

18. Waltenberger $\mathbf{J}$ (2001) Impaired collateral vessel development in diabetes: potential cellular mechanisms and therapeutic implications. Cardiovasc Res 49:554-560

19. Pala L, Ciani S, Dicembrini I et al (2010) Relationship between GLP-1 levels and dipeptidyl peptidase-4 activity in different glucose tolerance conditions. Diabet Med 27:691-695

20. Pala L, Mannucci E, Pezzatini A et al (2003) Dipeptidyl peptidase-IV expression and activity in human glomerular endothelial cells. Biochem Biophys Res Commun 310:28-31

21. Meneilly GS, Demuth HU, McIntosh CH et al (2000) Effect of ageing and diabetes on glucose-dependent insulinotropic polypeptide and dipeptidyl peptidase IV responses to oral glucose. Diabet Med 17:346-350

22. McKillop AM, Duffy NA, Lindsay JR et al (2008) Decreased dipeptidyl peptidase-IV activity and glucagon-like peptide-1(7-36) amide degradation in type 2 diabetic subjects. Diabetes Res Clin Pract 79:79-85

23. Christopherson KW 2nd, Hangoc G, Broxmeyer HE (2002) Cell surface peptidase CD26/dipeptidylpeptidase IV regulates CXCL12/stromal cell-derived factor-1 alpha-mediated chemotaxis of human cord blood $\mathrm{CD} 34^{+}$progenitor cells. J Immunol 169:7000-7008

24. Proost P, Struyf S, Schols D et al (1998) Processing by CD26/ dipeptidyl-peptidase IV reduces the chemotactic and anti-HIV-1 activity of stromal-cell-derived factor-1alpha. FEBS Lett 432:73-76

25. Zemani F, Silvestre JS, Fauvel-Lafeve F et al (2008) Ex vivo priming of endothelial progenitor cells with SDF-1 before transplantation could increase their proangiogenic potential. Arterioscler Thromb Vasc Biol 28:644-650

26. Sauve M, Ban K, Momen MA et al (2010) Genetic deletion or pharmacological inhibition of dipeptidyl peptidase-4 improves cardiovascular outcomes after myocardial infarction in mice. Diabetes 59:1063-1073

27. Zaruba MM, Theiss HD, Vallaster M et al (2009) Synergy between CD26/DPP-IV inhibition and G-CSF improves cardiac function after acute myocardial infarction. Cell Stem Cell 4:313-323

28. Fadini GP, Boscaro E, Albiero M et al (2010) The oral dipeptidyl peptidase-4 inhibitor sitagliptin increases circulating endothelial progenitor cells in patients with type 2 diabetes: possible role of stromal-derived factor-1alpha. Diabetes Care 33:1607-1609

29. Boulbou MS, Koukoulis GN, Makri ED et al (2005) Circulating adhesion molecules levels in type 2 diabetes mellitus and hypertension. Int J Cardiol 98:39-44

30. Nakamura K, Yamagishi S, Adachi H et al (2008) Serum levels of soluble form of receptor for advanced glycation end products (sRAGE) are positively associated with circulating AGEs and 
soluble form of VCAM-1 in patients with type 2 diabetes. Microvasc Res 76:52-56

31. Lambeir AM, Scharpe S, de Meester I (2008) DPP4 inhibitors for diabetes-what next? Biochem Pharmacol 76:1637-1643

32. Matheeussen V, Baerts L, de Meyer G et al (2011) Expression and spatial heterogeneity of dipeptidyl peptidases in endothelial cells of conduct vessels and capillaries. Biol Chem 392:189-198

33. Bjelke JR, Christensen J, Nielsen PF et al (2006) Dipeptidyl peptidases 8 and 9: specificity and molecular characterization compared with dipeptidyl peptidase IV. Biochem J 396:391399
34. Ajami K, Pitman MR, Wilson CH et al (2008) Stromal cellderived factors 1alpha and 1beta, inflammatory protein-10 and interferon-inducible T cell chemo-attractant are novel substrates of dipeptidyl peptidase 8. FEBS Lett 582:819-825

35. Aiello LP, Avery RL, Arrigg PG et al (1994) Vascular endothelial growth factor in ocular fluid of patients with diabetic retinopathy and other retinal disorders. $\mathrm{N}$ Engl $\mathrm{J}$ Med 331:1480-1487

36. Butler JM, Guthrie SM, Koc M et al (2005) SDF-1 is both necessary and sufficient to promote proliferative retinopathy. $\mathrm{J}$ Clin Invest 115:86-93 